\title{
Exacerbated Renal and Hematologic Toxicities to Ifosfamide and Doxorubicin-Based Chemotherapy in a Patient with Retroperitoneal Liposarcoma Harboring a Germline Mutation in the WRN Gene
}

\author{
Beatriz Mendes Awni ${ }^{a}$ Carlos Diego Holanda Lopesa \\ Guilherme Harada $^{a} \quad$ Alessandra Corte Real Salgues ${ }^{a}$

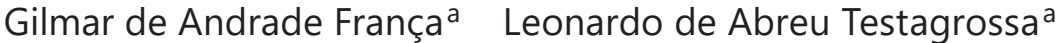 \\ Marcelo Araujo Queiroz ${ }^{a}$ b Adriano Gonçalves e Silva ${ }^{c}$ \\ Fabio de Oliveira Ferreirab Rodrigo Ramella Munhoz ${ }^{a}$, b \\ ${ }^{a}$ Oncology Center, Hospital Sírio-Libanês (HSL), São Paulo, Brazil; ${ }^{b}$ Cutaneous Malignancies \\ and Sarcomas Group, Hospital Sírio Libanês, São Paulo, Brazil; 'Cancer and Transplant \\ Institute, Curitiba, Brazil
}

\section{Keywords}

Werner's syndrome $\cdot$ WRN $\cdot$ Liposarcoma $\cdot$ Hematological and renal toxicities

\begin{abstract}
Werner's syndrome is caused by the inactivation of both WRN alleles and is characterized by premature aging and increased risk of neoplasms, especially those of mesenchymal origins, such as sarcomas. Given the characteristic genomic instability, patients with this syndrome are more susceptible to develop toxicities when exposed to cytotoxic agents, such as alkylators and anthracyclines. The impact of the monoallelic WRN mutation on treatment-associated toxicities is poorly understood. Here, we report a patient with locally advanced dedifferentiated liposarcoma of the retroperitoneum harboring a heterozygous germline inactivation mutation in the WRN gene, who was treated with a classic regimen of ifosfamide and doxorubicin and developed exacerbated and prolonged hematological and renal toxicities.
\end{abstract}




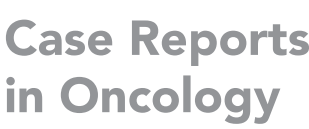

\begin{tabular}{l|l}
\hline Case Rep Oncol 2022;15:170-175 \\
\hline DOI: 10.1159/000521624 & $\begin{array}{l}\text { @ 2022 The Author(s). Published by S. Karger AG, Basel } \\
\text { www.karger.com/cro }\end{array}$ \\
\hline
\end{tabular}

Awni et al.: Chemotherapy Exacerbated Toxicities in a Patient with Germiline WRN Mutation

\section{Introduction}

The WRN gene codifies one of the five RecQ DNA helicase proteins and displays important functions in DNA replication and repair, as well as in the maintenance of telomeres and transcriptional modulation, through different DNA metabolic pathways [1]. Inactivation of both WRN alleles originates the Werner syndrome (WS), a rare autosomal recessive disease, characterized by premature aging and increased risk of various neoplasms, predominantly of mesenchymal origin, sarcomas being one of the most prevalent [2]. An overall incidence rate of less than 1 in 100,000 live births per year is estimated. Because of characteristically genomic instability, patients with WRN mutations may develop enhanced toxicities when exposed to cytotoxic agents, markedly alkylators and anthracyclines, such as ifosfamide and doxorubicin, which are frequently included in treatment regimens for patients with soft tissue sarcomas $[3,4]$. Nevertheless, the impact of heterozygous WRN mutation on treatmentassociated toxicities is uncertain. Here, we describe a case of a patient harboring a WRN mutation in heterozygosis with a locally advanced retroperitoneal dedifferentiated liposarcoma who developed exacerbated and prolonged hematological and renal toxicities after ifosfamide and doxorubicin exposure.

\section{Case Report}

A 60-year-old man with no comorbidities was diagnosed with a retroperitoneum mass, measuring $12 \times 12 \times 9 \mathrm{~cm}$ by magnetic resonance imaging, in July 2020 . The lesion biopsy was compatible with high-grade liposarcoma, and IHC stained positive for MDM2 and CDK4. From July 2020, he received systemic treatment with doxorubicin $25 \mathrm{mg} / \mathrm{m}^{2}$ and ifosfamide $3 \mathrm{~g} / \mathrm{m}^{2}$ on days 1-3 for four cycles. Throughout the systemic treatment performed at an outside facility, he required repeated transfusions of red blood cells due to grade 3 anemia and developed moderate to severe neutropenia. Myelotoxicity was accompanied by progressive worsening of renal function (Table 1). However, chemotherapy (CTx) dose adjustment was unnecessary because of a sustained creatinine clearance greater than $60 \mathrm{~mL} / \mathrm{min} / 1.73 \mathrm{~m}^{2}$ by Chronic Kidney Disease Epidemiology Collaboration. Restaging scans following cycle \#4 revealed a significant reduction in tumor size (Fig. 1), allowing for potential complete salvage surgery. Following the completion of CTx, the decision was made to proceed with neoadjuvant radiation therapy, performed to a total dose of $42 \mathrm{~Gy}$, delivered in 15 fractions. The patient then transitioned his care for surgical resection. Laboratory workup upon admission revealed both persistent myelotoxicity and renal insufficiency (creatinine clearance upon admission: $17 \mathrm{~mL} / \mathrm{min} / 1.73 \mathrm{~m}^{2}$ by Chronic Kidney Disease Epidemiology Collaboration ), despite an interval of 3 months since the last CTx cycle. Viral etiologies and nutritional deficiencies were ruled out. A myelogram was performed and showed normocellular bone marrow, with a maturation delay in the neutrophilic sector; a bone marrow biopsy showed no signs of malignancy. Concerning renal insufficiency workup, urinalysis revealed nonnephrotic proteinuria $(0.88 \mathrm{~g} / \mathrm{L})$ and glycosuria $(7.78 \mathrm{~g} / \mathrm{L})$; assessment of immune-mediated glomerulopathies was negative, and no significant morphological or vascular changes affecting the kidneys were identified through additional imaging. The genomic profiling by the hybridization-based protocol for germline assessment (Invitae, San Francisco, CA, USA) revealed a pathogenic heterozygous mutation in WRN (c.3123C>A; p.Cys1041) and variants of uncertain significance in WRN (c.4018C >T; p.Pro1340Ser), EGFR (c.2963A>G; p.His988Arg), RAD50 (c.2468G>A; p.Arg823Gln), and RECQL4 (c.2176G>A; p.Ala726Thr). The patient gradually recovered his blood counts until late December 2020; however, the renal function deteriorated to levels demanding renal replacement therapy (Table 1). He ultimately underwent

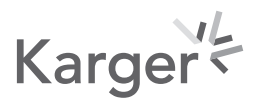


Table 1. Clinical laboratory results

\begin{tabular}{lllllll}
\hline & $\begin{array}{l}\text { Hemoglobin, } \\
\mathrm{g} / \mathrm{dL}\end{array}$ & $\begin{array}{l}\text { Leukocytes, } \\
\mathrm{g} / \mathrm{dL}\end{array}$ & $\begin{array}{l}\text { Neutrophils, } \\
\mathrm{g} / \mathrm{dL}\end{array}$ & $\begin{array}{l}\text { Platelets, } \\
/ \mathrm{mm} 3\end{array}$ & $\begin{array}{l}\text { Creatinine, } \\
\mathrm{mg} / \mathrm{dL}\end{array}$ & $\begin{array}{l}\text { Urea, } \\
\mathrm{mg} / \mathrm{dL}\end{array}$ \\
\hline 11 Apr 2019 & 14.8 & 6,060 & 4,266 & 200,000 & - & \\
19 Jun 2020 & 10.4 & 10,380 & 7,370 & 353,000 & - & \\
22 Jul 2020 (after 1st CTx) & 9.3 & 650 & - & 166,000 & - & \\
29 Aug 2020 & 10.1 & 9,810 & - & 303,000 & 0.96 & 30 \\
9 Sep 2020 (after 3rd CTx) & 9.1 & 470 & - & 143,000 & - & \\
19 Sep 2020 & 9.2 & 8,150 & 6,292 & 306,000 & 1.19 & 34 \\
29 Sep 2020 (after 4th CTx) & 8.8 & 240 & - & 172,000 & 1.89 & 40 \\
9 Dec 2020 & 8.3 & 840 & 20 & 121,000 & - & - \\
10 Dec 2020 & 8.6 & 1,070 & 30 & 105,000 & 3.8 & 76 \\
12 Dec 2020 & 8.0 & 1,100 & 40 & 115,000 & 3.98 & 88 \\
15 Dec 2020 & 7.3 & 2,270 & 970 & 115,000 & 3.72 & 77 \\
18 Dec 2020 & 7.8 & 5,690 & 4,450 & 107,000 & 4.27 & 83 \\
24 Dec 2020 & 8.5 & 4,870 & 3,910 & 135,000 & 5.29 & 99 \\
28 Dec 2020 & 9.1 & 2,910 & 2,140 & 153,000 & 6.02 & 103 \\
11 Jan 2021 & 9.0 & 4,290 & 3,140 & 165,000 & 7.80 & 105 \\
13 Jan 2021 & - & - & - & - & 8.38 & 107 \\
\hline
\end{tabular}

CTx, chemotherapy.

surgical resection of the lesion in the retroperitoneum, whose pathological report indicated a high-grade DDLPS (Fig. 2), and a left renal biopsy showed karyomegalic tubulointerstitial nephropathy (Fig. 3). He started continuous hemodialysis in the immediate postoperative period and was discharged in good clinical conditions after 6 weeks.

\section{Discussion}

Here, we report a patient with locally advanced DDLPS of the retroperitoneum and heterozygous germline inactivation mutation in the WRN gene, treated with a classic regimen of ifosfamide and doxorubicin and developed significant renal and hematologic toxicity. In mammalians, RecQ helicases comprise a group of 5 proteins (RECQL1, BLM, WRN, RECQL4, and RECQL5) involved in critical cellular processes, highlighting those associated with genome integrity maintenance. By using ATP hydrolysis energy, RecQ helicases unwind double-strand DNA and re-modulate secondary DNA structures and DNA-protein complexes, allowing those other processes to be activated to repair genome insults, for example [5]. The critical function of these helicases is represented in inheritable conditions characterized by genomic instability, exacerbated tissue injury to some biological or chemical agent when one of five proteins is lost or difunctional (e.g., Bloom syndrome and WS) [6].

WS is one of the classic progeroid syndromes characterized by premature aging and increased risk of neoplasms, predominantly of mesenchymal origin, and sarcomas are most prevalent. This is a rare condition with a global incidence rate of less than 1 in 100,000 live births per year [2]. The pathogenesis consists of a WRN locus loss of function in chromosome 8p12 with a difunctional RecQ helicase correspondent protein, causing rapid replicative senescence, chromosomal instability, and sensitivity to various agents that damage DNA $[7,8]$. 


\section{Case Reports in Oncology}

\begin{tabular}{l|l}
\hline Case Rep Oncol 2022;15:170-175 \\
\hline DOI: 10.1159/000521624 & $\begin{array}{l}\text { (c) 2022 The Author(s). Published by S. Karger AG, Basel } \\
\text { www.karger.com/cro }\end{array}$ \\
\hline
\end{tabular}

Awni et al.: Chemotherapy Exacerbated Toxicities in a Patient with Germiline WRN Mutation

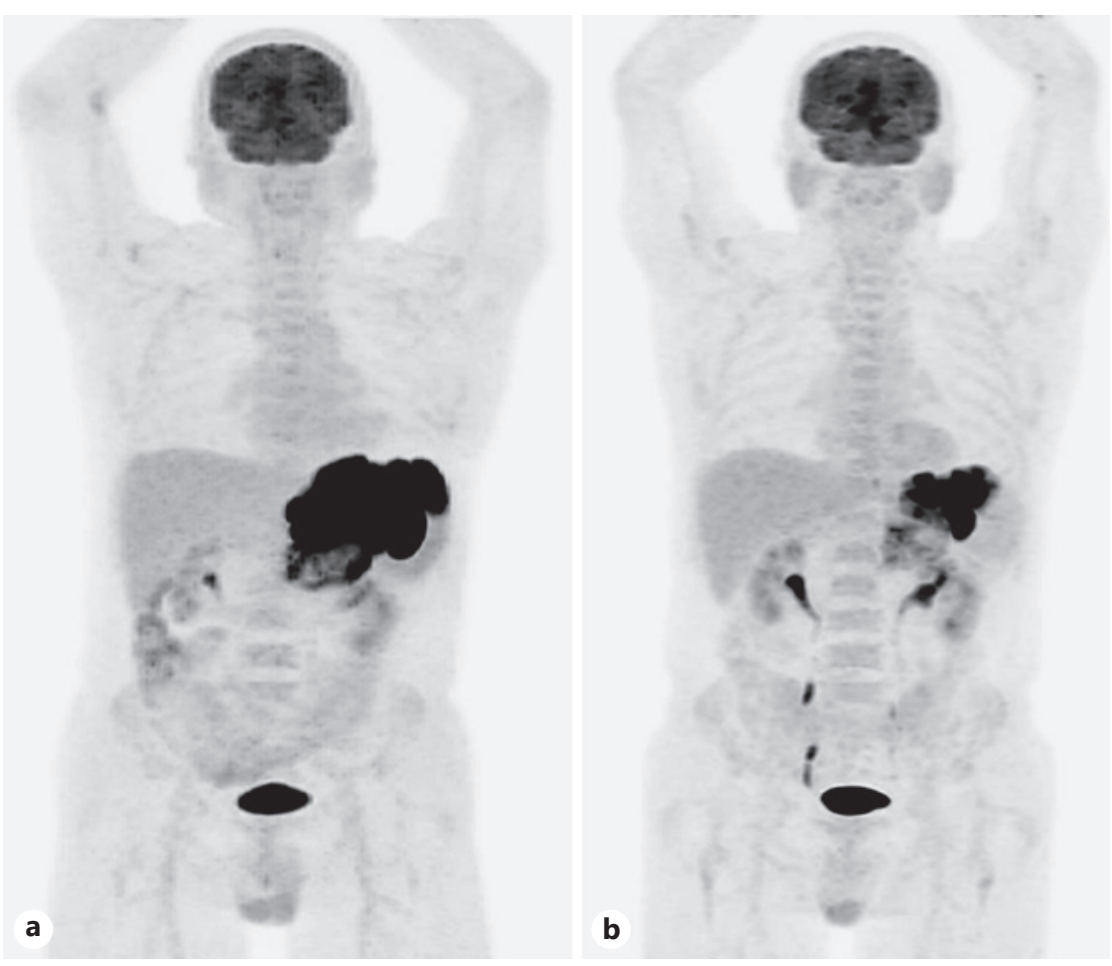

Fig. 1. 18-FDG PET scans showing areas of dedifferentiated liposarcoma of the retroperitoneum. a Baseline image demonstrating a heterogeneous mass with an infiltrative aspect and ill-defined borders in the retroperitoneum, in contact with the gastric wall and involving splenic artery, measuring $12.6 \times 12.8 \times 14.1 \mathrm{~cm}$, and a marked metabolism (SUVmax 37.0). b 18-FDG PET following neoadjuvant CTx with a reduction in the mass to $8.8 \times 6.6 \times 10.6 \mathrm{~cm}$ in the most significant axes and a slight reduction in the metabolic activity (SUVmax 34.7).
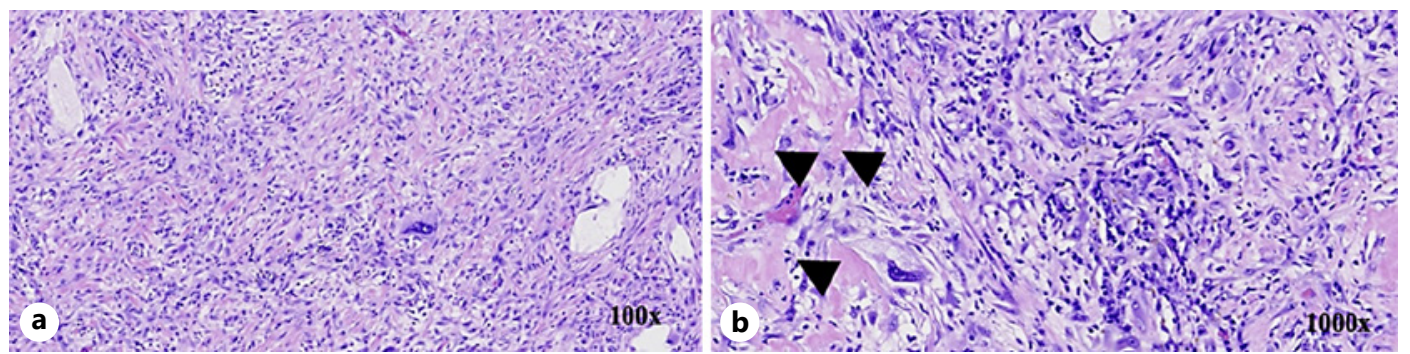

Fig. 2. H\&E stained photomicrographs of surgical specimen of DDLPS. $\mathbf{a}$, b Show dedifferentiated components of high-grade spindle cell sarcoma in about $100 \%$ of the tumor, high cellularity, necrosis present in $20 \%$ of the tumor (arrowheads), and 51 mitoses per 10 high-power fields. H\&E, hematoxylin and eosin

Despite the high incidence of sarcoma, there is little information regarding individualized treatment for patients with WS. Usually, a multimodal approach with anthracycline-based regimen CTx is the standard for localized soft tissue sarcomas [9]. In general, these drugs are well tolerated in cancer patients, but patients with mutations in the WRN gene may have more significant renal, neurological, cardiologic, and hematological toxicities [3, 4].

There are some reports of increased sensitivity of WS cells to the DNA damaged by cytotoxic CTx [10]. Moser et al. [11] used the glycophorin A somatic cell mutation assay to analyze genetic instability in vivo in patients with WS and heterozygotes, showing that both are 

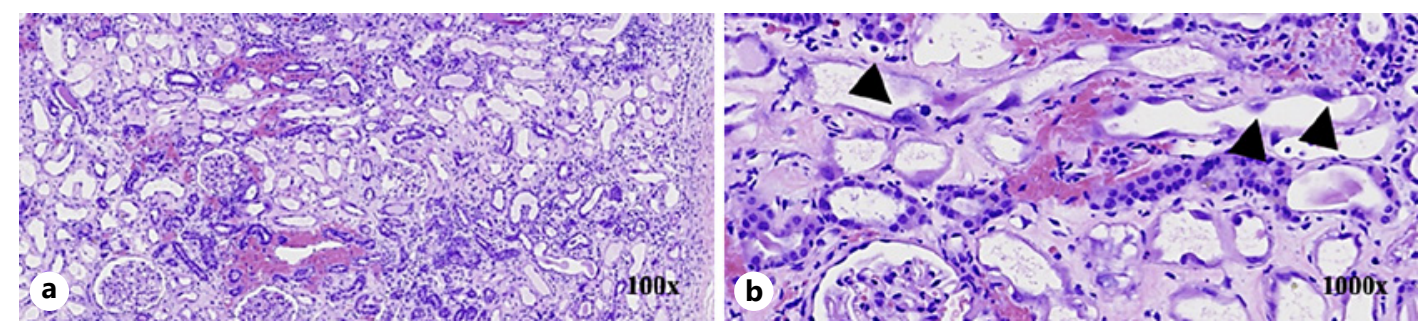

Fig. 3. H\&E stained photomicrographs of kidney biopsy. a, b Show diffusely dilated tubules with intensely regenerative epithelium and lymphoepithelial aggression. In some areas, the tubular epithelial nuclei are intensely enlarged (karyomegaly - arrowheads) and hyperchromatic presence of cell debris and neutrophils in light of some tubules. Interstice with diffuse edema and lymphoplasmacytic inflammatory infiltrate. H\&E, hematoxylin and eosin

heterozygous and homozygous mutation in WRN which had an increased risk of neoplasia and toxicities related to CTx. It was the first and only evidence published in the literature of genetic instability in patients with a monoallelic mutation in WRN.

Damage to renal tubular cells in settle of both defective DNA repair and increased toxicity to nephrotoxic agents can cause severe and irreversible nephritis, such as karyomegalic interstitial nephritis (KIN) [12]. This renal pathology is a rare cause of chronic hereditary interstitial nephritis. Generally, it presents as a slowly progressive chronic kidney disease, leading to end-stage kidney disease in early adulthood $[12,13]$. Although less common, ifosfamide-associated KIN has been described, mainly in young patients with a suggestion to recover renal function after a course of corticosteroids. Additionally, there is a direct association of mutations in Fanconi anemia-associated nuclease 1 (FAN1) as causing familiar KIN. As Fanconi anemia-associated nuclease 1 has endonuclease activity and is involved in DNA damage response in the Fanconi anemia pathway [14] and the RecQ helicases (like WRN) are involved in some of the primary mechanisms of DNA repair (base excision repair, nucleotide excision repair, and doublestrand break repair) [15], we postulated that WRN mutation present in this patient exacerbated the sensibility of cells to DNA-damaged agents, ifosfamide (an alkylating agent) and doxorubicin (a topoisomerase two inhibitor) reflecting in high-grade hematologic and renal toxicities.

\section{Conclusion}

To the best of our knowledge, this is the first case described in the literature showing a patient with a heterozygous mutation in WRN with dramatic renal and hematological toxicity after cytotoxic CTx. It is noteworthy that, in these patients, close monitoring of hematologic profile and renal function should proceed, and individualization of the best therapeutic strategy is essential to avoid severe toxicities.

\section{Statement of Ethics}

This case report was approved by the Ethics Committee of the Hospital Sírio-Libanês, São Paulo, Brazil. Written informed consent was obtained from the patient for publication of this case report and any accompanying images. 


\section{Conflict of Interest Statement}

The authors have no conflicts of interest to declare.

\section{Funding Sources}

No funding was received for this study.

\section{Author Contributions}

All authors contributed equally to this manuscript conception and writing.

\section{Data Availability Statement}

The data are not publicly available due to their containing information that could compromise the privacy of research participant.

\section{References}

1 Lebel M, Monnat RJ. Werner syndrome (WRN) gene variants and their association with altered function and age-associated diseases. Ageing Res Rev. 2018 Jan;41:82-97.

2 Oshima J, Sidorova JM, Monnat RJ. Werner syndrome: clinical features, pathogenesis and potential therapeutic interventions. Ageing Res Rev. 2017 Jan;33:105-14.

3 Seiter K, Qureshi A, Liu D, Galvin-Parton P, Arshad M, Agoliati G, et al. Severe toxicity following induction chemotherapy for acute myelogenous leukemia in a patient with Werner's syndrome. Leuk Lymphoma. 2005 Jul;46(7):1091-5.

4 Tsukamoto S, Kurematsu Y, Honoki K, Kido A, Somekawa S, Kaya D, et al. Severe toxicity of chemotherapy against advanced soft tissue sarcoma in Werner's syndrome: ifosfamide-induced encephalopathy with central diabetes insipidus. J Orthop Sci. 2016 May;21(3):403-6.

5 Bohr VA. Rising from the RecQ-age: the role of human RecQ helicases in genome maintenance. Trends Biochem Sci. 2008 Dec;33(12):609-20.

6 Larsen NB, Hickson ID. RecQ helicases: conserved guardians of genomic integrity. Adv Exp Med Biol. 2013; 767:161-84.

7 Yu C-E, Oshima J, Fu Y-H, Wijsman EM, Hisama F, Alisch R, et al. Positional cloning of the Werner's syndrome gene. Science. 1996 Apr 12;272(5259):258-62.

8 Gray MD, Shen J-C, Kamath-Loeb AS, Blank A, Sopher BL, Martin GM, et al. The Werner syndrome protein is a DNA helicase. Nat Genet. 1997 Sep;17(1):100-3.

9 von Mehren M, Kane JM, Bui MM, Choy E, Connelly M, Dry S, et al. NCCN guidelines insights: soft tissue sarcoma, version 1.2021: featured updates to the NCCN guidelines. J Natl Compr Canc Netw. 2020 Dec;18(12):1604-12.

10 Seiter K, Liu D, Feldman E, Shi Q, Qureshi A, Arshad M, et al. Long-term follow-up of high-dose mitoxantronebased induction therapy for patients with newly-diagnosed acute myelogenous leukemia. Twelve year results from a single institution. Leuk Lymphoma. 2006 Mar;47(3):425-32.

11 Moser MJ, Bigbee WL, Grant SG, Emond MJ, Langlois RG, Jensen RH, et al. Genetic instability and hematologic disease risk in Werner syndrome patients and heterozygotes. Cancer Res. 2000 May 1;60(9):2492-6.

12 Isnard P, Rabant M, Labaye J, Antignac C, Knebelmann B, Zaidan M. Karyomegalic interstitial nephritis: a case report and review of the literature. Medicine. 2016 May;95(20):e3349.

13 Burry AF. Extreme dysplasia in renal epithelium of a young woman dying from hepatocarcinoma. J Pathol. 1974 Jul;113(3):147-50.

14 Knipscheer P, Räschle M, Smogorzewska A, Enoiu M, Ho TV, Schärer OD, et al. The Fanconi anemia pathway promotes replication-dependent DNA interstrand cross-link repair. Science. 2009 Dec 18;326(5960):1698701.

15 Croteau DL, Popuri V, Opresko PL, Bohr VA. Human RecQ helicases in DNA repair, recombination, and replication. Annu Rev Biochem. 2014;83:519-52. 